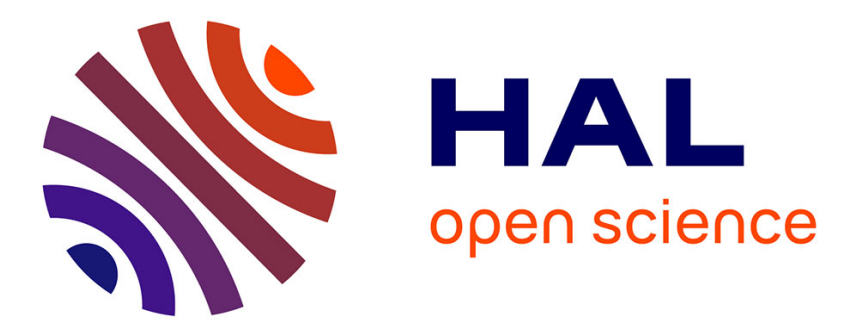

\title{
Hyperelastic Behaviour Identification by a Forward Problem Resolution: Application to a Tear Test of a Silicone-Rubber
}

\author{
M Giton, A. S. Caro-Bretelle, Patrick Ienny
}

\section{To cite this version:}

M Giton, A. S. Caro-Bretelle, Patrick Ienny. Hyperelastic Behaviour Identification by a Forward Problem Resolution: Application to a Tear Test of a Silicone-Rubber. Strain, 2006, 42 (4). hal03237528

\section{HAL Id: hal-03237528 \\ https://hal.mines-ales.fr/hal-03237528}

Submitted on 28 May 2021

HAL is a multi-disciplinary open access archive for the deposit and dissemination of scientific research documents, whether they are published or not. The documents may come from teaching and research institutions in France or abroad, or from public or private research centers.
L'archive ouverte pluridisciplinaire HAL, est destinée au dépôt et à la diffusion de documents scientifiques de niveau recherche, publiés ou non, émanant des établissements d'enseignement et de recherche français ou étrangers, des laboratoires publics ou privés. 


\title{
Hyperelastic Behaviour Identification by a Forward Problem Resolution: Application to a Tear Test of a Silicone-Rubber
}

\author{
M. Giton, A.-S. Caro-Bretelle and P. lenny \\ Ecole des Mines d'Alès, Centre des Matériaux de Grande Diffusion, 6, Av. de Clavières - 30319 Alès, France
}

\begin{abstract}
The mechanical behaviour of synthetic rubbers shows very high deformability, compressibility, time-dependent effect and strain softening. The present study is devoted to the analysis of local mechanical behaviour of silica-filled silicone rubber. New testing and identification are proposed in this paper by using standardised tear test, kinematic field measurements and a numerical inverse problem resolution to investigate localisation strain phenomena. The experimental procedure described hereafter, is based on strain field measurements using digital image processing. In-plane kinematic measurements by the digital image correlation are suitable to analyse nonhomogeneous mechanical tests performed especially on thin sheets: indeed, rubber-like materials are characterised by a very high deformability and a non-linear behaviour leading to important gradients of deformation. The identification procedure is conducted in two steps. First, parameters of the viscosity and stress softening (Mullins effect) are evaluated analytically by using axial and biaxial tensile tests. Then, hyperelastic parameters are identified by an inverse resolution based on standardised tear tests. The mechanical model is implemented into the finite element code Zebulon (Transvalor/ENSMP). The numerical model is built up by using informations on geometry and boundary conditions extracted from image sequence that were acquired during the test. Usage of different functions evaluating the distance between computed and experimental quantities (cost functions) in a minimisation process is discussed.
\end{abstract}

KEY WORDS: finite element analysis, mechanical testing, modelling, optical techniques, silica-filled silicone rubber

\section{NOTATION}

$\alpha$

Effective strain energy of the undamaged material attained up to time $t$

$\vec{b} \quad$ Sum of the nodal force values

$C_{10} \quad$ First coefficient characterizing rubber behaviour

$C_{01} \quad$ Second coefficient characterizing rubber behaviour

$C_{11} \quad$ Third coefficient characterizing rubber behaviour

$d_{\alpha} \quad$ Discontinuous damage

$d_{\alpha}^{\infty} \quad$ Maximum damage

$\eta_{\alpha} \quad$ Saturation value

$\mathbb{F} \quad$ Deformation gradient

$G(t)$ Shear modulus at time $t$

$G_{0} \quad$ Instantaneous strain shear modulus

$g_{i} \quad$ Relative modulus of term $i$ of Prony series

$\Gamma_{\mathrm{L}} \quad$ Free part of the boundary

$\Gamma_{\mathrm{U}} \quad$ Part of the boundary where displacements are prescribed

$\Gamma_{\mathrm{T}} \quad$ Part of the boundary where forces are prescribed
$I_{1} \quad$ First strain invariant

$I_{2} \quad$ Second strain invariant

J Gap function

$J_{T} \quad$ Gap function related to load

$J_{U} \quad$ Gap function related to displacement

$\overline{\bar{K}} \quad$ Stiffness matrix

$\Omega \quad$ Sample

$\partial \Omega \quad$ Boundary of the sample

$\vec{n} \quad$ Outward unit vector

$t \quad$ Time

$\vec{T} \quad$ Load applied on $\Gamma_{\mathrm{T}}$

$\tau_{i} \quad$ Relaxation time of term $i$ of Prony series

$\vec{u} \quad$ Displacement on $\Gamma_{\mathrm{U}}$

$\vec{S} \quad$ Sensitivity function

$W \quad$ Free strain energy

$W_{0} \quad$ Effective strain energy density function

$\vec{X} \quad$ Position of a particule in the reference configuration 


\section{Introduction}

Among various rubber materials, reinforced siliconerubbers exhibit both mechanical and physical properties that allow them to be used in many industrial applications. One of the most fundamental of them is related to the tear strength [1]. Geometries of the samples used in standardised tear tests conduct to a complex analyse of the stress concentration near de crack tip. Current researches, usually based on fracture mechanic analytical approach, require usage of stored energy functions to give a realistic approximation of the behaviour near the crack tips. Such a difficulty can be attributed to two main causes:

- The highly non-linear and inelastic behaviour of rubbers make difficult a correct estimation of the local response of the material.

- $\quad$ Standardised tear tests (ref NF-T46-007) provide a wide range of both heterogeneous strains and strain rates which are never reached for standard homogeneous tests.

A good understanding of the mechanical behaviour is necessary to reproduce a realistic response in a localisation area. The present paper deals with a testing procedure based on finite element analysis (FEA) and optical method combined to identify parameters of the mechanical behaviour model. This work follows upon a previous one where a viscous hyperelastic with damage model was proposed by using a direct approach [2-5]. The constitutive equation was iden- tified according to homogeneous tests. An optical instrumentation based on digital image correlation (DIC) provided macroscopic strain states.

The aim of the present work is to provide an experimental quantitative analysis of the high deformability and time-dependent effects near macroscopic strain concentrations. Experiment is based on a tensile test performed on standardised notched specimen (Figures 1 and 2). For this type of specimen, displacement field measurements based on image correlation technique [6] are used to provide experimental data (as displacements) particularly in the necking area. To answer this need, we used a version of the software of correlation specially adapted to the measure high strain $[3,4]$. A finite element model

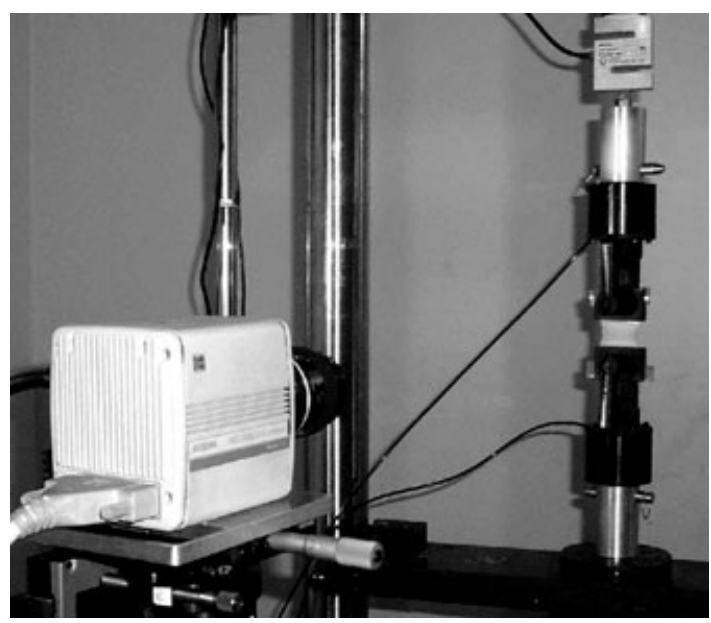

Figure I: Experimental set-up

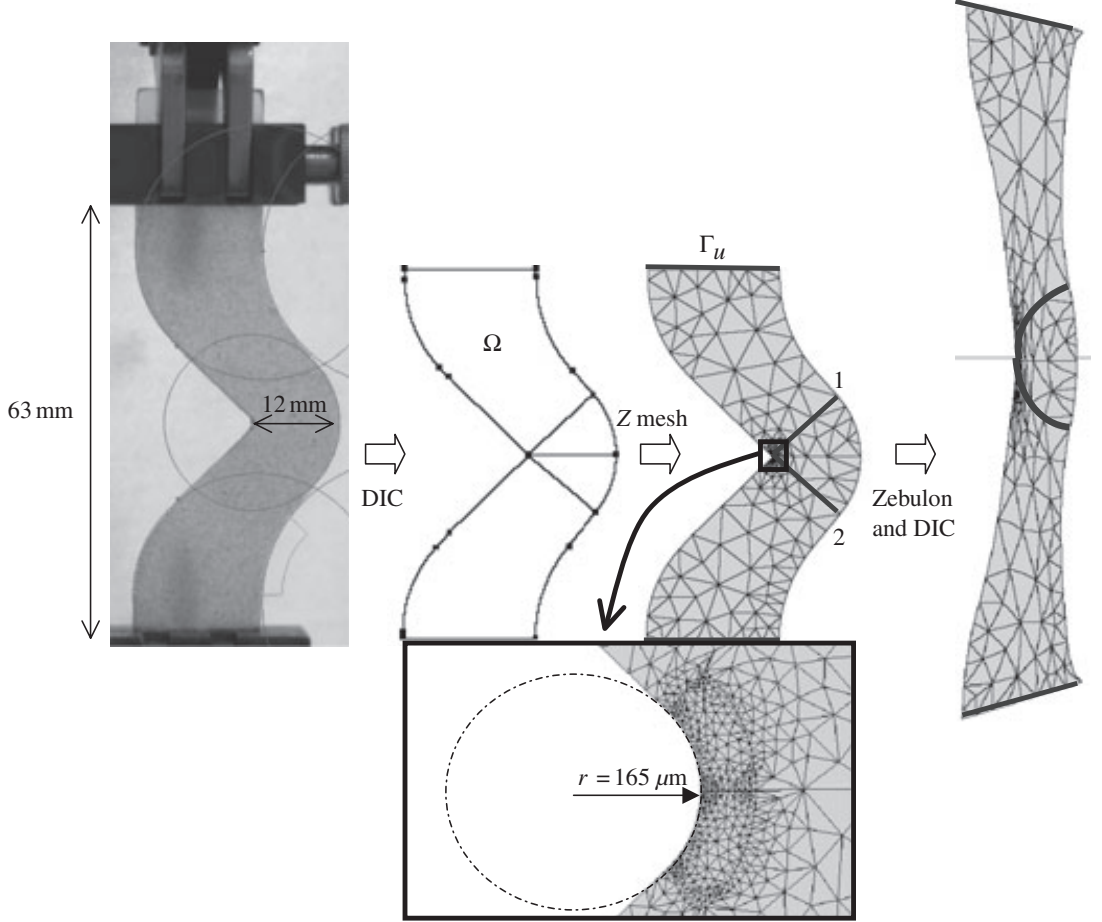

Figure 2: From Digital image recorded to finite element model: response of silicon-rubber under loading 
updating method (FEMU) is used more accurately to identify the parameters of the hyperelastic law. It consists of performing iteratively a FEA of the test so as to find constitutive parameters that achieve the best match between computed quantities (depending on the constitutive parameters) and their experimental counterparts. The interesting point in the solving process of the inverse problem concerns both boundary conditions and the geometry of the specimen which are provided by the optical method. The cost functions are based on displacements measured near the localisation area and the total tensile force.

The present work concentrates on the testing procedure to investigate the local mechanical behaviour near the crack tip. It includes the description of the tear test used to predict the response of the material in a local-gradient zone and the digital image processing which provides displacements fields. The constitutive parameters of the model behaviour are presented in the second section. Primarily, the set of parameters is identified from homogeneous tests (such as uniaxial or biaxial tensile tests) [2]. The third section presents the optimisation procedure based on FEA to identify parameters of mechanical behaviour. An FEM of the tear test is built up by using experimental data on geometry and boundary conditions. Cost functions are proposed and discussed in order to optimise the set of the material parameters.

\section{Experimental Analysis of the Tear Test}

Tear tests were performed on standardised specimen shaped in a rectangular sheet of silica fillers siliconerubber. Although the realistic geometry is measured by the optical method, the shape of the specimen and the stamped process were chosen to provide reproducible tests.

The standardised sample is located between the two jaws of a tensile machine (Figure 1). During the test we can follow both load submitted to the specimen and displacement of points of the sample. The displacement field measuring system was made of a CCD camera $(1024 \times 1024$ pixels, coded in 256 grey levels), an image acquiring system on a PC and a digital image processing. A sufficiently large part of the sample was recorded on the image to ensure the measurement of the boundary conditions during the test near the clamp even if the sample underwent large macroscopic strain. The optical axis of the camera remains perpendicular to the in-plane surface of the specimen due to the use of a three-axis rotational stage. During the mechanical test, images of the initial and deformed states were recorded.
In order to realise a finite element modelisation, realistic boundary conditions (RBC) give a realistic geometry of the specimen was achieved on the first image by Image Analysis (cinEMA, Era, France). This geometry defines the reference state of the undeformed specimen from which the outline and the mesh will be estimated (Figure 2). This process conducts to a realistic description of the sample including its asperities and artefacts.

Two-dimensional field of in-plane displacements were measured according the digital processing as mentioned above. Because of both large strain levels achieved in rubbers and the strain gradient combined with localisation phenomena, the processing used here is based on an iterative fine search method [3, 4]. The principle of the method is to recognise a point of a first image in the second image maximising a correlation function. This procedure allows reducing both the original number of intermediate images to achieve a given state and the correlation size for a better localisation measurement.

Otherwise, both displacement fields and distances relatives to the geometry of the specimen are given in pixels to avoid error from converting into metric unit. Thickness was only measured in metric units and then converted in pixels using the scale factor of images $(129 \mu \mathrm{m} / \mathrm{pixel})$. This scale factor represents a compromise between the sensitivity of displacement measurements and the minimisation of the out-of-plane effects [5]. The length between clamps was $63 \mathrm{~mm}$, the length of middle ligament is about $12 \mathrm{~mm}$ and the thickness is $1.5 \mathrm{~mm}$.

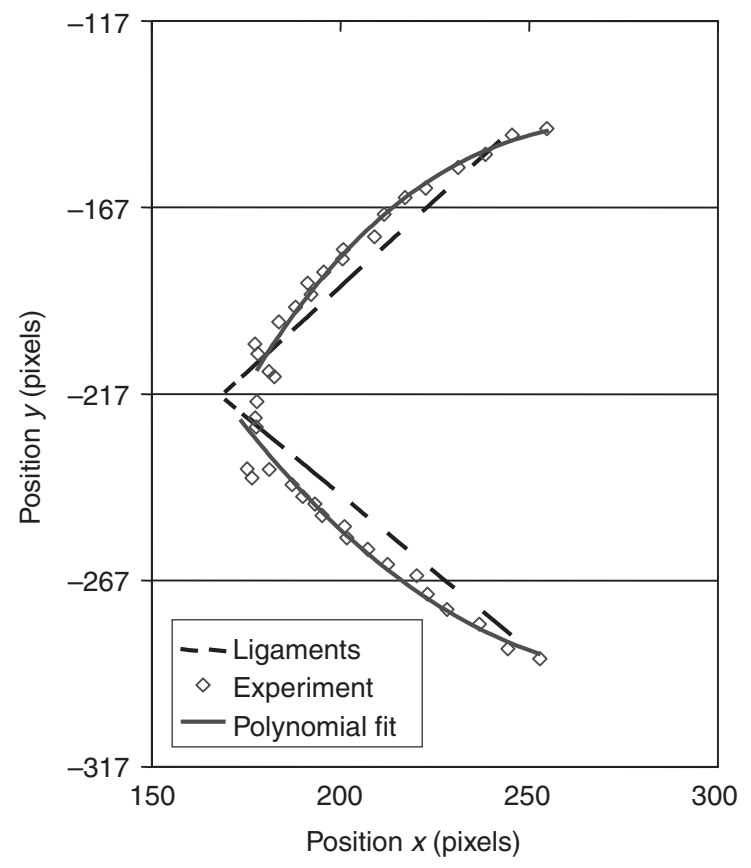

Figure 3: Nodal displacements determined by digital image correlation at time $46 \mathrm{~s}$ 
By this experimental analysis we can reach to the evolution of displacement for all nodes of the sample. For example if we focus our attention on the ligaments denoted 1 and 2 in Figure 2, we can follow at each step of the test the displacement of some nodes on these ligaments. To undisturbed the optimisation procedure by some possible errors (measurement), the nodal displacements measured by DIC on whole sections were approximated by a secondorder polynomial fit (Figure 3).

In this work the sensitivity of the technique with respect first to the unknown parameters and second to the error measurement will be discussed.

\section{The Phenomenological Constitutive Model}

The behaviour of synthetic rubbers is complex. Incompressibility, high deformability, time-dependent effect and strain softening are coupled. We opt for an incompressible hyperelastic law [7] including damage and viscosity. First of all, viscous and damage parameters are separately identified. Viscous parameters are analytically identified using a generalisation to large strain of the hereditary integral formulation [2]. The material is assumed to be isotropic and homogeneous. We represent the relaxation of the shear modulus $G(t)$ in terms of the Prony series as follows:

$\left.G(t)=G_{0} \quad g_{\infty}+\sum_{1}^{n} g_{i} e^{-t / \tau_{i}}\right) \quad$ with $g_{\infty}=1-\sum_{1}^{n} g_{i}$,

where $g_{i}$ and $\tau_{i}$ are the relative modulus and the relaxation time of terms $i$ respectively. $n$ is the number of non-zero terms used ( 8 for this application) and $G_{0}$ is the instantaneous strain shear modulus. Viscosity parameters are identified from homogeneous tests combined with a direct analytical resolution. According to damage accumulation generally observed in filled elastomers, a damage model was combined with the model described above. Following Miehe [8], the change in free energy, $W$, within an isothermal elastic deformation from the reference configuration to the current configuration is formally given by:

$W=\left(1-d_{\alpha}\right) W_{0} \quad$ with $d_{\alpha} \in[0,1]$

where $W_{0}$ refers to an effective strain energy density function with respect to undamaged material. Damage $d_{\alpha}$ is related to the so-called discontinuous damage and is written in the asymptotic form:

$d_{\alpha}(\alpha)=d_{\alpha}^{\infty}\left[1-\exp \left(-\frac{\alpha}{\eta_{\alpha}}\right)\right]$
Table I: Reference set of material parameters

\begin{tabular}{|c|c|c|c|c|c|c|c|c|}
\hline \multicolumn{9}{|c|}{ Linear viscosity } \\
\hline$g_{i}$ & 0.396 & 0.042 & 0.027 & 0.028 & 0.030 & 0.039 & 0.063 & 0.375 \\
\hline$\tau_{\mathrm{i}}(\mathrm{s})$ & $\infty$ & 20000 & 2282 & 400 & 60.00 & 9.0 & I.I & 0.14 \\
\hline \multicolumn{3}{|c|}{ Mullins effect } & \multicolumn{4}{|c|}{ Hyperelasticity } & & \\
\hline \multicolumn{2}{|l|}{$\eta_{\alpha}$} & $d_{\alpha}^{\infty}$ & \multicolumn{2}{|l|}{$C_{10}$} & \multicolumn{2}{|l|}{$\mathrm{C}_{01}$} & \multicolumn{2}{|c|}{$C_{11}$} \\
\hline \multicolumn{2}{|l|}{1.035} & 0.80 & \multicolumn{2}{|c|}{$2.72 \mathrm{MPa}$} & \multicolumn{2}{|c|}{$0.09 \mathrm{MPa}$} & \multicolumn{2}{|c|}{$0.40 \mathrm{MPa}$} \\
\hline
\end{tabular}

where $d_{\alpha}^{\infty}$ describes the maximum possible damage of the damage mechanism under consideration and $\eta_{\alpha}$ characterises the saturation property. Then, $\alpha$ define the effective strain energy of the undamaged material attained up to time $t$.

Secondly and according to Mooney [9] and Rivlin and Saunders [10], we characterise rubber behaviour by this strain density $W: \Omega \times \operatorname{Lin}^{+} \rightarrow \mathbb{R}$ such that:

$$
\begin{aligned}
W & (\vec{X}, \mathbb{F}) \\
& =C_{10}\left(I_{1}-3\right)+C_{01}\left(I_{2}-3\right)+C_{11}\left(I_{1}-3\right)\left(I_{2}-3\right) \\
& =\left(C_{10}, C_{01}, C_{11}\right)^{T} \cdot\left[\left(I_{1}-3\right),\left(I_{2}-3\right),\left(I_{1}-3\right)\left(I_{2}-3\right)\right]^{T} \\
& =\vec{C} \cdot \vec{I}
\end{aligned}
$$

where, $I_{1}$ and $I_{2}$ are the strain invariants depending on the deformation gradient $\mathbb{F}, \vec{X}$ designate the position of a particle in the reference configuration $\Omega$ and $\operatorname{Lin}^{+}$is this function space: $\operatorname{Lin}^{+}=$ $\{\overline{\bar{H}} \in \operatorname{Lin}: \operatorname{det}(\overline{\bar{H}})>0\}$. Lin is the space of all linear transformations from $\mathbb{R}^{3}$ into $\mathbb{R}$ and $T$ denotes the transpose.

From a latest research [2], we extracted a first system of material constants (Table 1).

\section{Finite Element Modelling of the Tear Specimen}

The non-homogeneous stress-strain state occurring for the structural mechanical test under study was analysed by using a finite element modelisation. Simulation were performed with a finite element analysis software (ZeBuLon Translator, ENSRP, France) by using digital data acquisition already described for the in-plane displacement measurements step. Image Analysis of the reference state provides outline of the mesh and realistic boundary conditions are estimated by DIC. Thus a virtual meshing was defined by using both geometry measured in reference image mentioned above and an interfacing with FEA software (ZeBuLon).

According to alignment defects and symmetric error measured by Image Analysis, the entire volume 
between horizontal sections has been meshed using 3D triangular 15-node linear elements. Undeformed and deformed meshing before fracture are shown in Figure 2. As we can see, the elements of the strain localisation zone are very distorted. This could be improved by using remeshing tools.

To ensure the correctness of numerical results, the sample is discretised into 1159 elements. Load or realistic displacements can be applied on part of the boundary. Solutions are computed in 71 time steps. The meshing corresponds to the further associated lists of points which define both horizontal sections near the clamps and transverse lines of points (1-2) on either side of the middle zone of the specimen. If the last list of measured points is at the source of the comparison presented below, the first one will define realistic boundary conditions.

\section{The Constitutive Equation Parameter Identification}

\section{Methodology}

Assuming the viscous and damage parameters known, we search for the hyperelastic behaviour. The lack of information about the strain density is compensated by additional data: digital image correlation gives field measurements either in the sample $(\Omega)$ or on its boundary $\partial \Omega$. For the model under study, $\partial \Omega$ is separated into $\Gamma$ and $\Gamma_{\mathrm{L}}\left(\partial \Omega=\Gamma \cup \Gamma_{\mathrm{L}}\right)$ and $\left.\Gamma \cap \Gamma_{\mathrm{L}}=\varnothing\right)$. We assume that the displacements $\vec{u}$ are prescribed on $\Gamma$ (Realistic Boundary Conditions by DIC), that the surface density forces $\vec{T}$ are known on $\Gamma$ (load cell) and that the surface $\Gamma_{\mathrm{L}}$ is free. In this problem $\Gamma=\Gamma_{\mathrm{U}}=\Gamma_{\mathrm{T}}$. A first simulation is conducted with the parameters introduced in Table 1 . Figure 4 shows for example the in-plane Lagrangian strains predicted by FEA at the middle ligament (at $46 \mathrm{~s}$ ). The elevated strain concentration factors at the equator of the notch can be noticed.

From the FEMU method used to enforce incompressibility - thanks to a comparison between computed quantities and their experimental counterparts - we were able to evaluate the constitutive parameter set. Two kinds of problem have been solved (see Table 2):

- The first problem is based on the deviation between experimental load applied on $\Gamma_{T}$ denoted $\vec{T}$ and the simulated one.

- The second problem adds a comparison on the deviation between measured and simulated displacements on two transverse lines 1 and 2 (Figure 2).

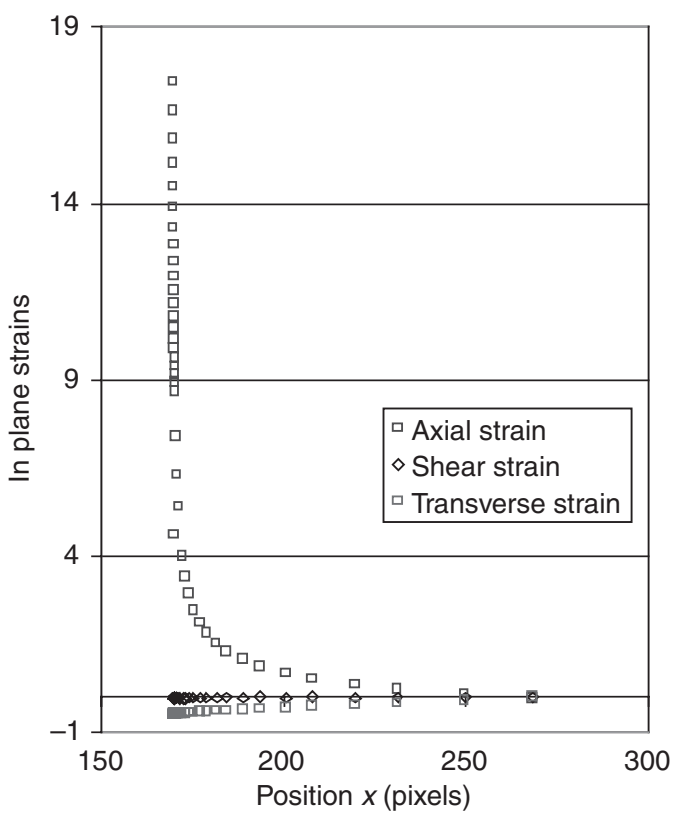

Figure 4: Strain components along the middle ligament at time $46 \mathrm{~s}$

Table 2: Known and unknown parameters for the resolution of the inverse problem

\begin{tabular}{lc}
\hline First problem & Second problem \\
\hline Known & Known \\
Geometry (DIC) & Geometry (DIC) \\
$u$ on $\Gamma_{u}($ RBC by DIC) & $u$ on $\Gamma_{u}(\mathrm{RBC}$ by DIC) \\
$T$ on $\Gamma_{T}$ (load cell) & $u$ in $\bar{\Omega}(\mathrm{DIC})$ \\
& $T$ on $\Gamma_{T}$ (load cell) \\
Damage parameters & Damage parameters \\
Viscous parameters & Viscous parameters \\
Unknown & Unknown \\
$C_{10}, C_{01}, C_{11}$ & $C_{10}, C_{01}, C_{11}$ \\
\hline
\end{tabular}

We opt for an optimisation based on Sequential Quadratic Programming algorithm. This secondorder local optimiser is included in the optimisation module 'Z-optim'. The gap between experimental and numerical values is computed via a cost function written as follow:

$J(\vec{C})=\frac{1}{2}[\vec{h}(\vec{C}, \varpi)-\vec{m}]^{2}$,

where $\vec{m}$ is the vector of the observed response from the experiments and $\vec{h}$ is the corresponding numerical information. $\vec{C}$ is the set of material parameters to extract. $\varpi$ represents data about geometry and boundaries conditions. For this study and to facilitate the interpretation of results, only hyperelastic parameters are identified.

For the first problem and with the notations introduced above, we define for each $\mathbb{F} \in \operatorname{Lin}^{+}$the functional $J_{T}: \mathbb{R}^{3} \rightarrow \mathbb{R}$ by: 
$J_{T}(\vec{C})=\frac{1}{2}\left(\overline{\frac{1}{\operatorname{det} \mathbb{F}} \frac{\partial W(\vec{X}, \mathbb{F})}{\partial \mathbb{F}} \mathbb{F}} \vec{n}-\vec{T}\right)^{2}$,

where $\vec{n}$ denotes the outward unit normal vector to the surface $\Gamma$.

The problem is thus to solve

$\inf J_{T}(\vec{C})$.

$\vec{C} \in \mathbb{R}^{3}$

It is a well-posed problem as $J_{T}$ is convex on $\mathbb{R}$. Indeed, let us rewrite $J_{T}$ as:

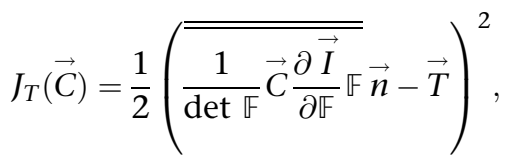

$\forall C_{1}, C_{2} \in \mathbb{R}^{3}$, one can compute the second directional derivative of $J_{T}$ :

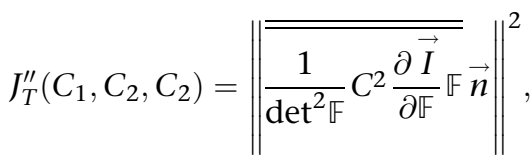

which is non-negative.

For the second problem, we add an optimisation on the displacements. After a finite element interpolation, the cost function in the minimisation takes the form:

$\inf _{\vec{C} \in \mathbb{R}^{3}} J(\vec{C})=\inf _{\vec{C} \in \mathbb{R}^{3}} J_{T}(\vec{C})+J_{U}(\vec{C}), \quad J_{U}(\vec{C})=\left[\overrightarrow{\bar{u}-K^{-1}(\vec{C})} \vec{b}\right]^{2}$,

where $\vec{k}$ and $\vec{b}$ are the stiffness matrix and the sum of nodal force value, respectively. As the stiffness matrix is not usually symmetric positive definite [11], the existence of such infrimum is not obvious.

\section{Results}

As illustrated in Figure 5 the optimisation via the first problem is in excellent agreement with experimental results. It improves the gap between experimental and simulated force obtained by the direct approach (Table 1). Figure 6 shows the evolution of axial displacements after a fit using both displacements and load corresponding to the second problem. It illustrates the difficulty to fit both the local behaviour (localisation) and the global one. In the case of this second optimisation, the displacement fit produces good results near the crack tips but overestimates the global surface density force.

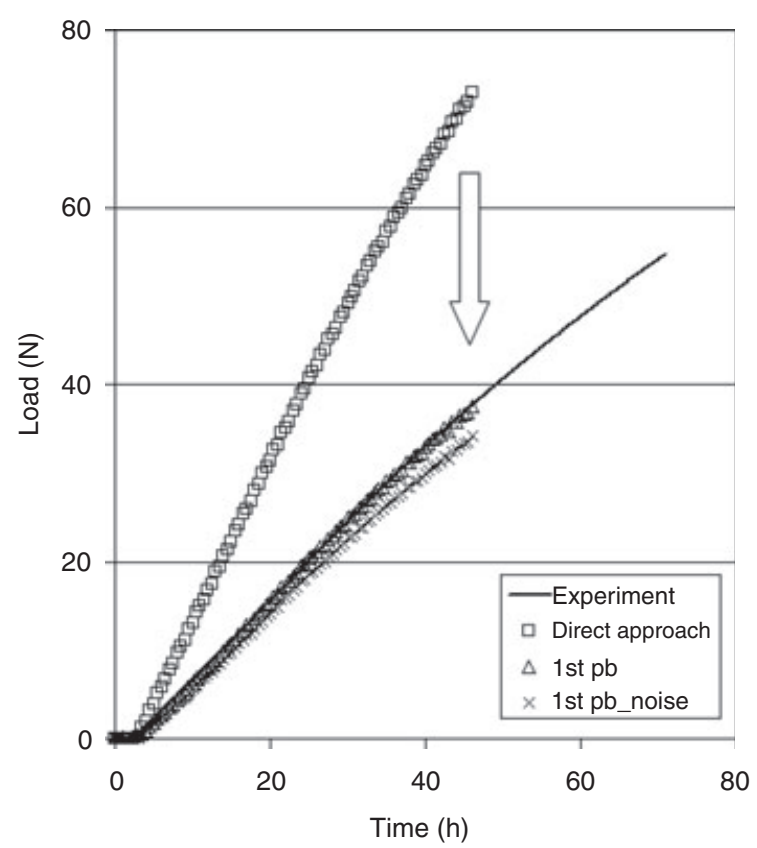

Figure 5: Load/time curves: experimental and simulation results

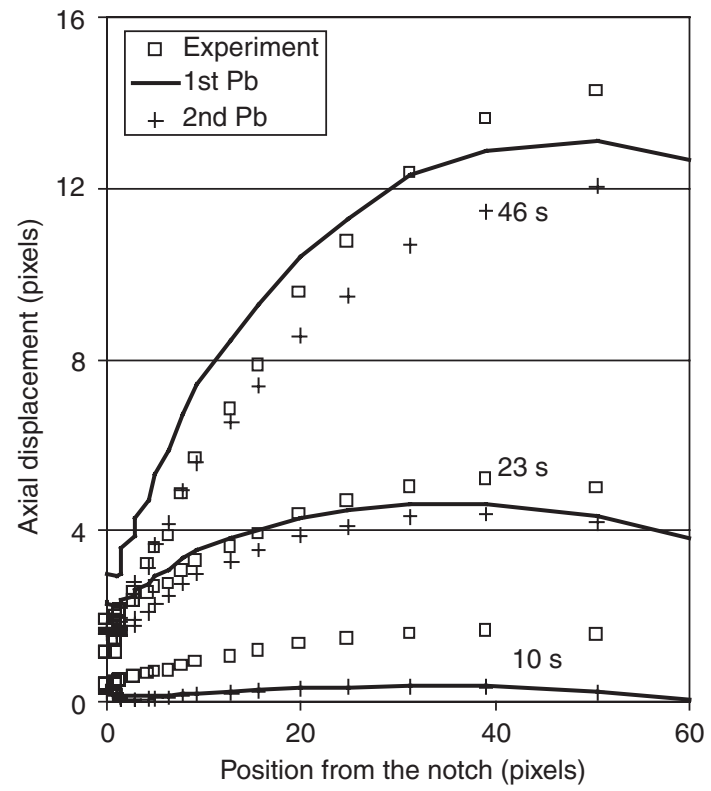

Figure 6: Nodal displacements along the superior transverse line

In order to assess this inverse procedure, we look for the sensitivity of experimental and numerical data with respect first to the surface density force and second to both surface density and displacements. A first simulation is then conducted with a perturbed prescribed displacement on $\Gamma$ (called 1st pb_noise). Comparable force results between $1 \mathrm{st} \mathrm{pb}$ and 1st pb_noise are obtained (Figure 5), DIC measurement errors can therefore be accepted. A second simulation slightly modified the hyperelastic coefficients (about $5 \%$ of their value). Adopting the notation of Methodology section, we write: 
Table 3: Sensitivity of the material set parameters on the cost function

\begin{tabular}{lllllll}
\hline \multicolumn{2}{l}{ First problem } & & & \multicolumn{3}{l}{ Second problem } \\
\cline { 1 - 3 } \cline { 5 - 6 } $\mathrm{C}_{10}$ & $\mathrm{C}_{01}$ & $\mathrm{C}_{11}$ & & $\mathrm{C}_{10}$ & $\mathrm{C}_{01}$ & $\mathrm{C}_{11}$ \\
\hline 0.93 & 1.79 & 1.78 & & 0.4650 & 0.895 & 0.89 \\
\hline
\end{tabular}

$\vec{S}=\left|\frac{\Delta J / \bar{J}}{\Delta \vec{C} / \overline{\vec{C}}}\right|$,

where $\Delta \theta / \bar{\theta}$ denotes the relative gap of the variable $\theta$. Table 3 shows the evaluation of sensitivity for each of the material parameters. We can notice a good homogeneity with respect to the parameters in the sensitivity results (Table 3 ).

\section{Conclusions}

In this study, we present a relevant methodology for the large strain mechanical behaviour characterisation of a silicone-rubber which combines two-dimensional in-plane displacement field measurements and finite element modelling. This protocol was applied on a structural component which is used to perform standardised tear test. With respect to the radius of the notch the level of the strain gradient at the equator is extremely large. This work provide an experimental quantitative field analysis of the stress concentration area and propose an identification procedure to calibrate a constitutive model that accounts for both global measured parameters as the load and the local response as displacement fields.

To describe the local response of the material, a special kinematic field measurements based on DIC have been developed. The FEM of the mechanical test is build according to both the real geometry of the specimen achieved by Image Analysis and realistic boundary conditions measured by DIC. The set of material parameters is extracted by using an optimisation scheme. Two kinds of cost functions were built. The first function accounts for the difference between measured and calculated load applied to the specimen. The second function associates in more a comparison on the basis of the measured displacements near the notch. If the last approach ensures realistic and reliable results in the strain concentration area, additional work is needed to discriminate between both optimisation techniques and cost functions used.

\section{REFERENCES}

1. Rivlin, R. S. and Thomas, A. G. (1953) Rupture of rubber part I: characteristic energy for tearing. J. Polym. Sci. 10, 291-318.

2. Giton, M., Ienny, P. and Piques, R. (2003) Viscous hyperelastic behaviour characterisation of a silicone-rubber: inverse problem resolution based on measurements by digital image correlation. COMPTEST 2003, Oral Communication, Châlons en Champagne.

3. Giton, M., Ienny, P. and Piques, R. (2001) Mesure de champs de déformation par corrélation d'images numériques: application à l'analyse locale en grande déformation des élastomères. GST Mécanique Expérimentale des Matériaux et des Structures et Environnement Mécanique, Oral Communication, Poitiers.

4. Giton, M. and Ienny, P. (2001) Amélioration des performances de la mesure des déformations par extensométrie optique bidimensionnelle. colloque Mecamat, Aussois: 196199.

5. Laraba-Abbes, F., Ienny, P. and Piques, R. (2003) A new 'tailor-made' methodology for the mechanical behaviour analysis of rubber-like materials: I. Kinematics measurements using a digital speckle extensometry. Polymer 44, 807-820.

6. Sutton, M. A., Wolters, W. J., Peters, W. H., Ranson, W. F. and Mcneill, S. R. (1983) Determination of displacements using an improved digital correlation method. Image Vis. Comput. 1, 133-139.

7. Holzapfel, G. A. (2000) Non linear Solid Mechanics, a Continuum Approach for Engineering. John Wiley and Sons, Chichester.

8. Miehe, C. (1995) Discontinuous and continuous damage evolution in Ogden-type-large-strain elastic materials. Eur. J. Mech. A Solids 14, 697-720.

9. Mooney, M. (1940) A theory of large elastic deformation. J. Appl. Phys. 11, 582-592.

10. Rivlin, R. S. and Saunders, D. W. (1951) Large elastic deformation of isotropic materials VII, experiments on the deformation of rubber. Phil. Trans. R. Soc. Lond. A243, 251-288.

11. Ponthot, J. P. (1994) Traitement unifié de la Mécanique des Milieux Continus solides en grandes transformations par la méthode des éléments finis. Thèse. Université de Lièges, Belgium. 